\title{
Gender-Dependent Effect of GSTM1 Genotype on Childhood Asthma Associated with Prenatal Tobacco Smoke Exposure
}

\author{
Chih-Chiang Wu, ${ }^{1,2,3}$ Chia-Yu Ou, ${ }^{4}$ Jen-Chieh Chang, ${ }^{5}$ Te-Yao Hsu, ${ }^{4}$ \\ Ho-Chang Kuo, ${ }^{6}$ Chieh-An Liu, ${ }^{7}$ Chih-Lu Wang, ${ }^{7}$ Chia-Ju Chuang, ${ }^{2}$ Hau Chuang, ${ }^{5}$ \\ Hsiu-Mei Liang, ${ }^{4}$ and Kuender D. Yang ${ }^{2,3}$ \\ ${ }^{1}$ Department of Pediatrics, Show Chwan Memorial Hospital, 542 Section 1, Zhongshan Road, Changhua 50008, Taiwan \\ ${ }^{2}$ Department of Medical Research, Show Chwan Medical System in Chang Bing, 6 Lu-Kung Road, Changhua 50544, Taiwan \\ ${ }^{3}$ Institute of Clinical Medicine, National Yang-Ming University, 155 Section 2, Linong Street, Taipei 11221, Taiwan \\ ${ }^{4}$ Department of Obstetrics and Gynecology, Kaohsiung Chang Gung Memorial Hospital, \\ Taiwan and Chang Gung University College of Medicine, 123 Dapi Road, Kaohsiung 83301, Taiwan \\ ${ }^{5}$ Genomic and Proteomic Core Laboratory, Department of Medical Research, Kaohsiung Chang Gung Memorial Hospital and \\ Chang Gung University College of Medicine, 123 Dapi Road, Kaohsiung 83301, Taiwan \\ ${ }^{6}$ Department of Pediatrics, Kaohsiung Chang Gung Memorial Hospital and Chang Gung University College of Medicine, \\ 123 Dapi Road, Kaohsiung 83301, Taiwan \\ ${ }^{7}$ Department of Pediatrics, Po-Jen Hospital, 350 Bo'ai 2nd Road, Kaohsiung 81358, Taiwan
}

Correspondence should be addressed to Kuender D. Yang; yangkd.yeh@hotmail.com

Received 5 June 2014; Accepted 19 August 2014; Published 18 September 2014

Academic Editor: Carla M. Calò

Copyright ( $) 2014$ Chih-Chiang Wu et al. This is an open access article distributed under the Creative Commons Attribution License, which permits unrestricted use, distribution, and reproduction in any medium, provided the original work is properly cited.

It remains unclear whether the GSTM1 genotype interacts with tobacco smoke exposure (TSE) in asthma development. This study aimed to investigate the interactions among GSTM1 genotype, gender, and prenatal TSE with regard to childhood asthma development. In a longitudinal birth cohort in Taiwan, 756 newborns completed a 6-year follow-up, and 591 children with DNA samples available for GSTM1 genotyping were included in the study, and the interactive influences of gender-GSTM1 genotypingprenatal TSE on childhood asthma development were analyzed. Among these 591 children, 138 (23.4\%) had physician-diagnosed asthma at 6 years of age, and 347 (58.7\%) were null-GSTM1. Prenatal TSE significantly increased the prevalence of childhood asthma in null-GSTM1 children relative to those with positive GSTM1. Further analysis showed that prenatal TSE significantly increased the risk of childhood asthma in girls with null-GSTM1. Furthermore, among the children without prenatal TSE, girls with null-GSTM1 had a significantly lower risk of developing childhood asthma and a lower total IgE level at 6 years of age than those with positive GSTM1. This study demonstrates that the GSTM1 null genotype presents a protective effect against asthma development in girls, but the risk of asthma development increases significantly under prenatal TSE.

\section{Introduction}

The prevalence of childhood asthma has increased worldwide in recent decades [1]. Environmental factors, including increasing air pollution, tobacco smoke exposure, a lower load of infection with pathogens, increased use of industrial materials in buildings, urbanization, and certain nutritional factors, may play an important role in this evolving epidemic.
Recently, increasing evidence has demonstrated that certain types of environmental exposure may increase the risk of asthma development for certain genetic backgrounds [2], implying that the gene-environment interaction is critical in asthma development.

Oxidative stress has been implicated in the pathogenesis of asthma, which is characterized by chronic airway inflammation. The glutathione S-transferases (GSTs) are a family 
of enzymes that have the general function of detoxifying xenobiotics that are capable of generating free radicals, by conjugating them with glutathione. GSTM1 has been extensively studied because its locus is polymorphic with a common null allele that produces a complete lack of the enzyme. The association between the GSTM1 null genotype and asthma development is not well established in the current literature. Several studies have demonstrated an increased risk of asthma or decreased lung function in subjects with the GSTM1 null genotype [3-9], whereas other studies have reported no association between the GSTM1 genotype and asthma [10-12]. The results of systematic reviews and metaanalyses of the effects of GSTM1 on asthma are also controversial. Some studies have revealed that the GSTM1 null genotype significantly increases the risk of asthma in children and adults $[13,14]$. One meta-analysis showed that the GSTM1 null genotype may be associated with an increased risk of asthma (pooled OR 1.28; 95\% CI 1.09-1.52), with large between-study heterogeneity. However, the association disappeared when the meta-analysis was repeated for the largest nine studies [15]. Another meta-analysis found no significant association between the GSTM1 polymorphism and asthma [16]. Several studies investigating the geneenvironment interaction with regard to asthma development found that environmental oxidative stresses, such as tobacco smoke exposure [17-19] and ozone [20, 21], increased the risk of asthma in children with the GSTM1 null genotype but not in those with positive GSTM1. Another study showed that maternal use of acetaminophen in late pregnancy increased the risk of asthma or wheezing in children when the maternal or child's GSTM1 genotype was positive [22]. Functional studies on the role of GSTM1 in asthma are limited. Our previous studies have demonstrated that gene-gene and geneenvironment interactions for $\mathrm{IgE}$ production begin in the prenatal stage [23-25]. This study aimed to investigate the effect of the GSTM1 genotype on the relationships among prenatal tobacco smoke exposure (TSE), childhood asthma development, and allergic sensitization for different gender backgrounds in a longitudinal birth cohort study in Southern Taiwan.

\section{Methods}

2.1. Study Design and Subjects. To study the effect of genegene and gene-environment interactions on prenatal and postnatal IgE production and the development of allergic diseases, a longitudinal birth cohort study was conducted at Kaohsiung Chang Gung Memorial Hospital, Taiwan, as reported previously [23-25]. In this cohort, the parents of 1848 children were prenatally recruited by our study nurse to enroll the birth cohort. Among the 1848 children, 1629 children were born in the hospital. In total, 1546, 1348, 1236, and 756 of the 1629 children completed the 6-month, 18month, 3-year, and 6-year follow-up visits, respectively. DNA samples collected at the newborn stage (from the umbilical cord blood) and at 6 years of age were subjected to GSTM1 genotyping in this study. The study protocol was approved by the Institutional Review Board, and informed consent was provided to the parents at the prenatal stage. The information regarding parental atopy history and family smoking habits was obtained from a questionnaire administered during prenatal recruitment. We defined the infants as having prenatal TSE if any family member at home had a habit of smoking indoors at home. The atopy history of the children, including atopic dermatitis, allergic rhinitis, or asthma if ever diagnosed by a physician, was acquired from the questionnaire given to parents at the 6-year follow-up. Cord blood samples were collected immediately after the infant's birth for DNA collection. Blood samples were collected from children at the 6-year follow-up for DNA extraction and the measurement of allergen sensitization through the detection of specific IgE levels in response to egg whites (f1), cow's milk (f2), peanuts (f13), shrimp (f24), house dust mites (d1), and German cockroaches (i6) (Phadia CAP system) because house dust mites (approximately 90\%) and German cockroaches, rather than pollen or mold (both $<2 \%$ ), are the major aeroallergens of children with asthma in Taiwan [26].

2.2. Analysis of GSTM1 Polymorphisms. Blood leukocytes were subjected to DNA extraction using the Gentra Puregene kit (Qiagen Inc., Valencia, CA) and then stored at $-80^{\circ} \mathrm{C}$ after $70 \%$ alcohol precipitation.

The genetic polymorphism analysis for the GSTM1 genes was performed using an individual multiplex PCR approach [27] with the following primers: $\mathrm{F} 5^{\prime}$-CGCCATCTTGTGCTACATTGCCCG- $3^{\prime}$ and R $5^{\prime}$-TTCTGGATTGTAGCAGATCA-3' for GSTM1 and F $5^{\prime}$-CAACTTCATCCACGTTCACC- $3^{\prime}$ and R $^{\prime}$-GAAGAGCCAAGGACAGGTAC- $3^{\prime}$ for $\beta$-globin. Briefly, the multiplex PCR was performed in a $25 \mu \mathrm{L}$ reaction mixture consisting of $50 \mathrm{ng}$ of DNA, $2.5 \mu \mathrm{L}$ of $10 \times$ GenTaq buffer, $10 \mathrm{mM}$ dNTP mix, each primer at $10 \mu \mathrm{M}$, and $1 \mathrm{U}$ of GenTaq DNA polymerase. The PCR reaction was performed in a PCR thermal cycler system (Applied Biosystems, Life Technologies) with an initial denaturation at $95^{\circ} \mathrm{C}$ for $5 \mathrm{~min}$ and then $35 \mathrm{cycles}$ of $50 \mathrm{~s}$ at $94^{\circ} \mathrm{C}, 50 \mathrm{~s}$ at $60^{\circ} \mathrm{C}$, and $50 \mathrm{~s}$ at $72^{\circ} \mathrm{C}$, followed by a final elongation for $10 \mathrm{~min}$ at $72^{\circ} \mathrm{C}$. The amplified products were visualized on a $2 \%$ agarose gel. The GSTM1 genotypes were determined by the presence or absence of bands at $230 \mathrm{bp}$, with a $260 \mathrm{bp}$ internal control ( $\beta$-globin). Through this simple multiplex PCR approach, the null and positive genotypes for GSTM1 could be clearly identified, although heterozygous and homozygous positive genotypes could not be differentiated.

2.3. Data Analysis and Statistics. The demographic data for the children with or without GSTM1 genotypes and the prevalence of allergic diseases and allergic sensitization at 6 years of age in children with or without prenatal TSE were analyzed using the chi-squared test. Multiple logistic regressive analyses were performed for childhood asthma with several factors, including preterm, gender, paternal and maternal atopy, and prenatal TSE. The log-transformed IgE levels at 6 years of age among the groups were analyzed by one-way ANOVA and Bonferroni post hoc tests. 


\section{Results}

3.1. Demographic Data for the Birth Cohort with 6-Year Follow-Up. Of the cohort of 756 children who completed the follow-up at 6 years of age, 591 subjects, whose DNA samples collected as newborns and at 6 years of age were available, were subjected to GSTM1 genotyping in this study. Among these 591 subjects, 244 (41.3\%) were positive and 347 (58.7\%) were null for the GSTM1 genotype. In total, 188 (31.8\%), $320(54.1 \%)$, and 138 (23.4\%) of these children had been diagnosed at 6 years of age with atopic dermatitis, rhinitis, or asthma, respectively, by a physician. In total, 579 of the 591 children received an allergic sensitization test at the 6year follow-up, and 256 (43.3\%) children were sensitized to house dust mites. Among the 591 children analyzed in this study, children with prenatal TSE had a significantly higher risk of developing asthma than children without prenatal TSE ( $P=0.006$, OR: $1.780,95 \%$ CI: 1.181-2.684). However, there were no significant differences in the development of atopic dermatitis or rhinitis between children with and without prenatal TSE (Table 1). Moreover, there were no differences in food allergen sensitization or house dust mite sensitization between these 2 groups (data not shown).

\subsection{Prenatal TSE Increases the Risk of Childhood Asthma in} Null-GSTM1 Subjects Compared to Positive GSTM1 Children. Next, the relationship between prenatal TSE and childhood asthma in children with the GSTM1 null or positive genotype was analyzed. Prenatal TSE significantly increased the prevalence of childhood asthma in children with the GSTM1 null genotype ( $P=0.002$, OR: $2.337,95 \% \mathrm{CI}: 1.370-3.985)$ compared with those with positive GSTM1 $(P=0.550)$ (Table 1). Furthermore, a multivariate logistic regression analysis of childhood asthma in children with the GSTM1 null or positive genotype was performed to adjust for other demographic data, such as gender, prematurity, and maternal and paternal atopy. In the multivariate logistic regression analysis, male gender and prenatal TSE both significantly increased the prevalence of childhood asthma in children with the GSTM1 null genotype, whereas prenatal TSE was not a significant risk factor in children with positive GSTM1 (Table 2).

3.3. Gender-Dependent Association between GSTM1 Null Genotype and Asthma. To analyze the association between prenatal TSE and childhood asthma for the GSTM1 null and positive genotypes and in different gender backgrounds, we categorized all of the children into four groups (males with positive GSTM1 genotype, males with GSTM1 null genotype, females with positive GSTM1 genotype, and females with GSTM1 null genotype). Prenatal TSE significantly increased the risk of childhood asthma in females with the GSTM1 null genotype $(30.0 \%$ versus $9.4 \%, P=0.001$, OR: 4.107 , 95\% CI: 1.699-10.107) compared with the effect in the other 3 groups (Table 3 and Figure 1). Furthermore, among children without prenatal TSE, females with the GSTM1 null genotype had a significantly lower risk of developing childhood asthma compared with females with positive GSTM1 (9.4\% versus

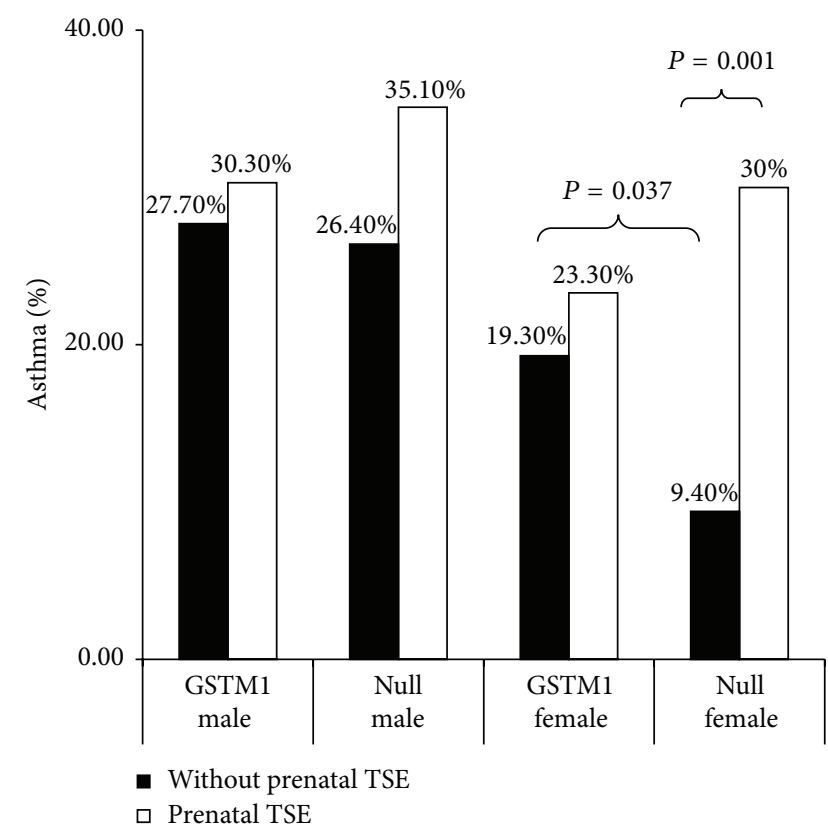

FIGURE 1: Interactions among GSTM1 genotype, gender, and prenatal TSE with regard to asthma development at 6 years of age. Prenatal TSE significantly increased the risk of childhood asthma in females with the GSTM1 null genotype ( $P=0.001$, OR: $4.107,95 \%$ CI: $1.669-$ 10.107), but not in the other 3 groups. Furthermore, among children without prenatal TSE, females with the GSTM1 null genotype had a significantly lower risk of developing childhood asthma $(P=0.036$, OR: 0.436, 95\% CI: 0.197-0.966).

19.3\%, $P=0.037$, OR: 0.436, 95\% CI: 0.197-0.966) (Table 3 and Figure 1).

3.4. Gender-Dependent Association between GSTM1 Null Genotype and Total IgE Levels. Among the children without prenatal TSE, the log-transformed total IgE level at 6 years of age was significantly lower in female children with the GSTM1 null genotype than in the other 3 groups (Figure 2). Additionally, male and female children with the GSTM1 null genotype presented lower house dust mite and food allergen sensitization compared with those with positive GSTM1, but the difference was not significant (Table 4).

\section{Discussion}

This study demonstrates that the GSTM1 null genotype could have a bipolar effect on childhood asthma development, depending on gender and prenatal TSE. In particular, the GSTM1 null genotype is a protective factor against asthma in girls without prenatal TSE but becomes a risk factor for asthma with prenatal TSE.

Asthma is a chronic inflammatory airway disorder associated with airway hyperresponsiveness and reversible airflow limitation in response to specific triggers. Disturbances in oxidation/reduction (redox) reactions and impaired antioxidant defenses have been associated with asthma [28], and lower systemic GSH levels and higher GSSG levels have been 
TABLE 1: Allergic diseases in children with or without prenatal TSE at 6 years of age ( 6 y) and with different GSTM1 genotypes.

\begin{tabular}{|c|c|c|c|c|c|}
\hline & Prenatal TSE & No prenatal TSE & $P$ & OR & $95 \% \mathrm{CI}$ \\
\hline $6 \mathrm{y}$ dermatitis & $48 / 156$ & $140 / 435$ & 0.745 & 0.937 & $0.631-1.390$ \\
\hline GSTM1 null & $30 / 95$ & $77 / 252$ & 0.854 & 1.049 & $0.631-1.745$ \\
\hline GSTM1 positive & $18 / 61$ & $63 / 183$ & 0.480 & 0.797 & $0.425-1.496$ \\
\hline 6 y rhinitis & $90 / 156$ & $230 / 435$ & 0.300 & 1.215 & $0.840-1.758$ \\
\hline GSTM1 null & $60 / 95$ & $134 / 252$ & 0.095 & 1.510 & $0.930-2.451$ \\
\hline GSTM1 positive & $30 / 61$ & $96 / 183$ & 0.657 & 0.877 & $0.491-1.566$ \\
\hline $6 y$ asthma & $49 / 156$ & $89 / 435$ & 0.006 & 1.780 & $1.181-2.684$ \\
\hline GSTM1 null & $32 / 95$ & $45 / 252$ & 0.002 & 2.337 & $1.370-3.985$ \\
\hline GSTM1 positive & $17 / 61$ & $44 / 183$ & 0.550 & 1.221 & $0.634-2.348$ \\
\hline
\end{tabular}

TABLE 2: Multivariate regression analysis of childhood asthma in children with the GSTM1 null or positive genotype.

\begin{tabular}{|c|c|c|c|c|c|c|}
\hline & \multicolumn{3}{|c|}{ GSTM1 null } & \multicolumn{3}{|c|}{ GSTM1 positive } \\
\hline & $P$ & OR & $95 \% \mathrm{CI}$ & $P$ & OR & $95 \% \mathrm{CI}$ \\
\hline Male gender & 0.003 & 2.317 & $1.342-4.000$ & 0.126 & 1.606 & $0.875-2.950$ \\
\hline Preterm ( $<37$ weeks) & 0.583 & 0.725 & $0.230-2.285$ & 0.723 & 0.784 & $0.205-2.999$ \\
\hline Maternal atopy $^{\dagger}$ & 0.488 & 1.235 & $0.681-2.240$ & 0.595 & 1.206 & $0.604-2.409$ \\
\hline Paternal atopy $^{\dagger}$ & 0.228 & 1.419 & $0.803-2.509$ & 0.125 & 1.643 & $0.871-3.101$ \\
\hline Prenatal TSE & 0.003 & 2.308 & $1.333-3.997$ & 0.553 & 1.223 & $0.629-2.377$ \\
\hline
\end{tabular}

${ }^{\dagger}$ Atopy is defined by phenotypic asthma, allergic rhinitis, or atopic dermatitis along with a detectable serum specific $\operatorname{IgE}(\geq 0.35 \mathrm{kU} / \mathrm{L})$ response to one or more common allergens (egg white, cow's milk, peanut, shrimp, house dust mite, or German cockroach).

TABLE 3: Association of prenatal TSE and childhood asthma in individuals of different genders and GSTM1 genotypes.

\begin{tabular}{|c|c|c|c|c|c|c|}
\hline \multicolumn{7}{|c|}{ Childhood asthma } \\
\hline & & $\begin{array}{c}\text { No TSE } \\
\text { Asthma number/total } \\
\text { number (percentage) }\end{array}$ & $\begin{array}{c}\text { Prenatal TSE } \\
\text { Asthma number/total } \\
\text { number (percentage) }\end{array}$ & \multirow[t]{2}{*}{$P$} & \multirow[t]{2}{*}{ OR } & \multirow[t]{2}{*}{$95 \% \mathrm{CI}$} \\
\hline & & OR (95\% CI) & OR (95\% CI) & & & \\
\hline \multirow{2}{*}{ Male } & \multirow{2}{*}{ GSTM1 } & 28/101 (27.7\%) & $10 / 33(30.3 \%)$ & 0.775 & 1.134 & $0.479-2.681$ \\
\hline & & 1.000 (reference) & 1.000 (reference) & & & \\
\hline \multirow{2}{*}{ Male } & \multirow{2}{*}{ Null } & $32 / 129(26.4 \%)$ & 20/57 (35.1\%) & 0.227 & 1.510 & $0.773-2.952$ \\
\hline & & $0.933(0.519-1.676)$ & $1.243(0.495-3.121)$ & & & \\
\hline \multirow{2}{*}{ Female } & \multirow{2}{*}{ GSTM1 } & 17/88 (19.3\%) & $7 / 30(23.3 \%)$ & 0.637 & 1.271 & $0.469-3.448$ \\
\hline & & 1.000 (reference) & 1.000 (reference) & & & \\
\hline \multirow{2}{*}{ Female } & \multirow{2}{*}{ Null } & 12/127 (9.4\%) & $12 / 40(30.0 \%)$ & 0.001 & 4.107 & $1.669-10.107$ \\
\hline & & $0.436(0.197-0.966)$ & $1.408(0.477-4.159)$ & & & \\
\hline
\end{tabular}

TABLE 4: The GSTM1 null genotype presented a lower prevalence of house dust mite sensitization and food allergen sensitization compared with positive GSTM1 in boys and girls without prenatal TSE, but the difference was not significant.

\begin{tabular}{lcccccccccc}
\hline & \multicolumn{2}{c}{ Male } & & & \multicolumn{3}{c}{ Female } \\
& \multicolumn{2}{c}{ GSTM1 } & \multicolumn{2}{c}{ Null } & $P$ & GSTM1 & Null & \multicolumn{2}{c}{$P$} \\
\hline 6 y HDM sensitization $^{*}$ & $51 / 100$ & $51.0 \%$ & $57 / 126$ & $45.2 \%$ & 0.389 & $37 / 87$ & $42.5 \%$ & $48 / 122$ & $39.3 \%$ & 0.644 \\
6 y food allergen sensitization $^{*}$ & $27 / 100$ & $27.0 \%$ & $31 / 126$ & $24.6 \%$ & 0.682 & $22 / 87$ & $25.3 \%$ & $23 / 122$ & $18.9 \%$ & 0.265 \\
6 y any sensitization $^{\dagger}$ & $59 / 100$ & $59.0 \%$ & $66 / 126$ & $52.4 \%$ & 0.32 & $42 / 87$ & $48.3 \%$ & $52 / 122$ & $42.6 \%$ & 0.418 \\
\hline
\end{tabular}

${ }^{*} \mathrm{HDM}$ sensitization is defined by a specific IgE response to house dust mites $(\mathrm{d} 1) \geqq 0.35 \mathrm{kU} / \mathrm{L}$.

${ }^{\#}$ Food allergen sensitization is defined by a detectable serum specific $\operatorname{IgE}(\geq 0.35 \mathrm{kU} / \mathrm{L})$ response to one or more food allergens (egg white, cow's milk, peanut, or shrimp).

${ }^{\dagger}$ Any sensitization is defined by a detectable serum specific $\operatorname{IgE}(\geq 0.35 \mathrm{kU} / \mathrm{L})$ response to one or more common allergens (egg white, cow's milk, peanut, shrimp, house dust mite, or German cockroach). 


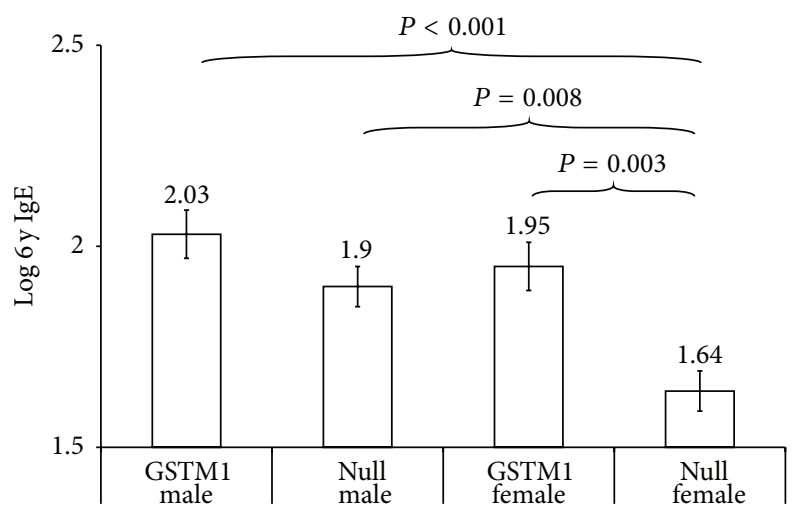

FIGURE 2: Interaction of the GSTM1 genotype and gender with regard to the log-transformed total IgE level at 6 years of age among individuals without prenatal TSE. Among children without prenatal TSE, the log-transformed total IgE level at 6 years of age was significantly lower in females with the GSTM1 null genotype than in the other 3 groups. The results are presented as the mean \pm standard error.

reported to be related to asthma development and increased asthma severity [29-31]. GSTM1, one of the most studied enzymes in the GST family, functions in the detoxification of xenobiotics, environmental toxins, and products of oxidative stress by conjugation with glutathione, thereby protecting cells from reactive oxygen species (ROS). Individuals with the GSTM1 null genotype lose GSTM1 enzymatic activity and thus may be vulnerable to oxidative stress in the airway and have a higher risk for airway inflammation. Most previous studies have reported that the GSTM1 null genotype significantly increases the risk for asthma development, although some studies found this risk to be insignificant. Wu et al. found that stimulation of primary human bronchial epithelial cells (positive GSTM1) with diesel exhaust particles (DEP) significantly increased IL- 8 and IL- $1 \beta$ protein expression, and knockdown of GSTM1 in these cells further elevated DEP-induced IL- 8 and IL- $1 \beta$ expression, implying that GSTM1 deficiency aggravates DEP-induced proinflammatory responses [32].

Similar to a previous study showing that the GSTM1 null genotype increases the risk of childhood asthma only in children with a history of intrauterine smoke exposure [33], this study found that the GSTM1 null genotype is associated with a higher incidence of asthma than the positive GSTM1 genotype is in boys and girls with prenatal TSE, with a nonsignificant difference $35.3 \%$ versus $30.3 \%, P=$ 0.643 , OR: 1.243 , $95 \%$ CI: $0.495-3.121$ for boys, $30.0 \%$ versus $23.3 \%, P=0.535$, OR: $1.408,95 \%$ CI: $0.477-4.159$ for girls). Additionally, we found that prenatal TSE increases the risk of childhood asthma in individuals with the GSTM1 null genotype, which supports the findings of other studies [1719]. Furthermore, we found that prenatal TSE significantly increases the risk of childhood asthma in girls with the GSTM1 null genotype, but not in boys with the GSTM1 null genotype.

Among the children without prenatal TSE, girls with the GSTM1 null genotype had a significantly lower incidence of asthma and lower log-transformed IgE level at 6 years of age than girls with positive GSTM1. Additionally, among individuals (either boys or girls) without prenatal TSE, the GSTM1 null genotype was associated with lower house dust mite or food allergen sensitization, but the difference was not significant. These results suggest that the GSTM1 null genotype may protect against the development of asthma in girls if no prenatal TSE is present. This hypothesis is supported by a study in which the GSTM1 null genotype is associated with a decreased risk for asthma among atopic subjects [34] and another study investigating the effect of the positive and null-GSTM1 genotypes on allergeninduced oxidant stress, airway inflammation, and reactivity in vivo in adults with mild atopic asthma and without a regular asthma medication [35]. In this study, patients with a positive genotype had higher baseline and allergenprovoked airway neutrophilia and higher concentrations of myeloperoxidase than GSTM1 null patients. The allergenstimulated generation of the acute-stress and proneutrophilic mediators tumor necrosis factor- $\alpha$, CXCL- 8 , IL- $1 \beta$, and IL6 and the postallergen airway concentrations of IgE and the neutrophil-generated mediators matrix metalloproteinase- 9 , B-cell activating factor, transforming growth factor- $\beta 1$, and elastase were also higher in patients with positive GSTM1. This study also found that GSTM1 positive individuals with asthma were more reactive to specific allergens, producing a $20 \%$ decrease in FEV1, but eosinophil inflammation and allergeninduced F2-isoprostane levels, which are considered to be specific markers of oxidative stress in vivo, were unaffected. These findings imply that certain phenotypes of asthma may be affected by the GSTM1 genotype.

There are certain limitations in this study. Not all children who completed the 6-year follow-up had DNA samples qualified for GSTM1 genotyping, making selection bias possible. However, the demographic data on the 591 and 165 children with and without available DNA samples, respectively, including data on parental allergic diseases and sensitization, gender, prematurity, and the presence or absence of prenatal TSE, were not significantly different (data not shown). Moreover, our study examined TSE at the prenatal stage but not in the infant stage or childhood stage. Furthermore, we acknowledge that caution must be used in generalizing these results to other populations, because the GST loci strongly interact with the environment. The different environmental risk factors, which are present in human populations, may certainly influence the results of this type of genetic association studies.

This study yields additional evidence for the geneenvironment interaction with regard to the development of childhood asthma and IgE level, starting in the prenatal stage. Based on the present findings, the GSTM1 null genotype in girls may be a protective factor against childhood asthma development and IgE level. However, girls with the GSTM1 null genotype are much more vulnerable to environmental oxidative stresses, such as prenatal TSE. The mechanism may not involve simple redox equilibrium because the GSTM1 genotype (null or positive) was previously found to be unrelated to the total plasma GST enzymatic activity, and 
the lack of GSTM1 activity for the GSTM1 null genotype may be compensated by other members of the GST superfamily [36]. Similarly, another study also found that positive GSTM1 was possibly related to neutrophilic inflammation in asthma without affecting allergen-induced $\mathrm{F} 2$-isoprostane levels [35]. Further studies are necessary to investigate the exact mechanism by which the GSTM1 genotype influences airway inflammation and allergic responses.

\section{Practical Implications}

This study demonstrates a gene-environment-gender interaction in the development of childhood asthma. Specifically, prenatal TSE increases the prevalence of childhood asthma. The GSTM1 null genotype is a protective factor against asthma development in girls without prenatal TSE but becomes a risk factor with prenatal TSE.

\section{Disclosure}

Chih-Chiang Wu and Chia-Yu Ou are joint first authors.

\section{Conflict of Interests}

The authors declare that they have no conflict of interests.

\section{Acknowledgments}

This study was supported in part by funding from Grants NSC 97-2314-B-182A-054-MY2 and NSC98-3112-B-182-004 (97IR007) from the National Science Council of Taiwan; Grants CMRPG860422, CMRPG850353, and CZRPG880253 from the Chang Gung Memorial Hospital, Taiwan; and Grant CMRPRA11024 from the Show Chwan Memorial Hospital, Taiwan. The authors thank the staff of the delivery room and outpatient clinics of Kaohsiung Chang Gung Memorial Hospital, Kaohsiung, Taiwan, for the collection of blood samples in this cohort study. The authors acknowledge the technical support provided by the Genomic \& Proteomic Core Laboratory, Department of Medical Research, Kaohsiung Chang Gung Memorial Hospital.

\section{References}

[1] W. Maziak, T. Behrens, T. M. Brasky et al., "Are asthma and allergies in children and adolescents increasing? Results from ISAAC phase I and phase III surveys in Münster, Germany," Allergy, vol. 58, no. 7, pp. 572-579, 2003.

[2] M. W. Su, C. H. Tsai, K. Y. Tung et al., "GSTP1 is a hub gene for gene-air pollution interactions on childhood asthma," Allergy, vol. 68, no. 12, pp. 1614-1617, 2013.

[3] T. Islam, K. Berhane, R. McConnell et al., "Glutathione-Stransferase (GST) P1, GSTM1, exercise, ozone and asthma incidence in school children," Thorax, vol. 64, no. 3, pp. 197-202, 2009.

[4] T. Ivaschenko, O. Sideleva, and V. Baranov, "Glutathione-Stransferase $\mu$ and theta gene polymorphisms as new risk factors of atopic bronchial asthma," Journal of Molecular Medicine, vol. 80, no. 1, pp. 39-43, 2002.
[5] M. Saadat, I. Saadat, Z. Saboori, and A. Emad, "Combination of CC16, GSTM1, and GSTT1 genetic polymorphisms is associated with asthma," Journal of Allergy and Clinical Immunology, vol. 113, no. 5, pp. 996-998, 2004.

[6] F. D. Gilliland, W. James Gauderman, H. Vora, E. Rappaport, and L. Dubeau, "Effects of glutathione-S-transferase M1, T1, and P1 on childhood lung function growth," The American Journal of Respiratory and Critical Care Medicine, vol. 166, no. 5, pp. 710716, 2002.

[7] L. I. Holla, A. Stejskalova, and A. Vasku, "Polymorphisms of the GSTM1 and GSTT1 genes in patients with allergic diseases in the Czech population," Allergy: European Journal of Allergy and Clinical Immunology, vol. 61, no. 2, pp. 265-267, 2006.

[8] R. A. Karam, H. F. Pasha, A. S. El-Shal, H. M. A. Rahman, and D. M. Gad, "Impact of glutathione-S-transferase gene polymorphisms on enzyme activity, lung function and bronchial asthma susceptibility in Egyptian children," Gene, vol. 497, no. 2, pp. 314-319, 2012.

[9] C. S. P. Lima, I. A. Néri, G. J. Lourenço, I. C. J. Faria, J. D. Ribeiro, and C. S. Bertuzzo, "Glutathione S-transferase mu 1 (GSTM1) and theta 1 (GSTT1) genetic polymorphisms and atopic asthma in children from Southeastern Brazil," Genetics and Molecular Biology, vol. 33, no. 3, pp. 438-441, 2010.

[10] S. Piacentini, A. Verrotti, R. Polimanti et al., "Functional polymorphisms of GSTA1 and GSTO2 genes associated with asthma in Italian children," Clinical Chemistry and Laboratory Medicine, vol. 50, no. 2, pp. 311-315, 2012.

[11] P. Reddy, R. N. Naidoo, T. G. Robins et al., "GSTM1 and GSTP1 gene variants and the effect of air pollutants on lung function measures in South African children," American Journal of Industrial Medicine, vol. 55, no. 12, pp. 1078-1086, 2012.

[12] S. Piacentini, R. Polimanti, B. Moscatelli, M. Re, D. Manfellotto, and M. Fuciarelli, "Lack of association between GSTM1, GSTP1, and GSTT1 gene polymorphisms and asthma in adult patients from Rome, central Italy," Journal of Investigational Allergology and Clinical Immunology, vol. 22, no. 4, pp. 252-256, 2012.

[13] S. Liang, X. Wei, C. Gong et al., "Significant association between asthma risk and the GSTM1 and GSTT1 deletion polymorphisms: an updated meta-analysis of case-control studies," Respirology, vol. 18, no. 5, pp. 774-783, 2013.

[14] F. Li, S. Li, H. Chang et al., "Quantitative assessment of the association between the GSTM1-Null genotype and the risk of childhood asthma," Genetic Testing and Molecular Biomarkers, vol. 17, no. 9, pp. 656-661, 2013.

[15] C. Minelli, R. Granell, R. Newson et al., "Glutathione-Stransferase genes and asthma phenotypes: a Human Genome Epidemiology (HuGE) systematic review and meta-analysis including unpublished data," International Journal of Epidemiology, vol. 39, no. 2, Article ID dyp337, pp. 539-562, 2010.

[16] S. Piacentini, R. Polimanti, I. Simonelli et al., "Glutathione S-transferase polymorphisms, asthma susceptibility and confounding variables: a meta-analysis," Molecular Biology Reports, vol. 40, no. 4, pp. 3299-3313, 2013.

[17] F. D. Gilliland, Y.-F. Li, L. Dubeau et al., "Effects of glutathione S-transferase M1, maternal smoking during pregnancy, and environmental tobacco smoke on asthma and wheezing in children," The American Journal of Respiratory and Critical Care Medicine, vol. 166, no. 4, pp. 457-463, 2002.

[18] M. Kabesch, C. Hoefler, D. Carr, W. Leupold, S. K. Weiland, and E. von Mutius, "Glutathione $S$ transferase deficiency and passive smoking increase childhood asthma," Thorax, vol. 59, no. 7, pp. 569-573, 2004. 
[19] C. N. A. Palmer, A. S. F. Doney, S. P. Lee et al., "Glutathione $S$-transferase M1 and P1 genotype, passive smoking, and peak expiratory flow in asthma," Pediatrics, vol. 118, no. 2, pp. 710716, 2006.

[20] I. Romieu, M. Ramirez-Aguilar, J. J. Sienra-Monge et al., "GSTM1 and GSTP1 and respiratory health in asthmatic children exposed to ozone," European Respiratory Journal, vol. 28, no. 5, pp. 953-959, 2006.

[21] I. Romieu, J. J. Sienra-Monge, M. Ramírez-Aguilar et al., "Genetic polymorphism of GSTM1 and antioxidant supplementation influence lung function in relation to ozone exposure in asthmatic children in Mexico City," Thorax, vol. 59, no. 1, pp. $8-10,2004$.

[22] S. O. Shaheen, R. B. Newson, S. M. Ring, M. J. Rose-Zerilli, J. W. Holloway, and A. J. Henderson, "Prenatal and infant acetaminophen exposure, antioxidant gene polymorphisms, and childhood asthma," The Journal of Allergy and Clinical Immunology, vol. 126, no. 6, pp. 1141.e7-1148.e7, 2010.

[23] C.-A. Liu, C.-L. Wang, H. Chuang, C.-Y. Ou, T.-Y. Hsu, and K. D. Yang, "Prenatal prediction of infant atopy by maternal but not paternal total IgE levels," The Journal of Allergy and Clinical Immunology, vol. 112, no. 5, pp. 899-904, 2003.

[24] K. D. Yang, J.-C. Chang, H. Chuang et al., "Gene-gene and geneenvironment interactions on IgE production in prenatal stage," Allergy: European Journal of Allergy and Clinical Immunology, vol. 65, no. 6, pp. 731-739, 2010.

[25] K. D. Yang, C.-Y. Ou, T.-Y. Hsu et al., "Interaction of maternal atopy, CTLA-4 gene polymorphism and gender on antenatal immunoglobulin E production," Clinical and Experimental Allergy, vol. 37, no. 5, pp. 680-687, 2007.

[26] K.-S. Wan, W. Yang, and W.-F. Wu, "A survey of serum specificIge to common allergens in primary school children of taipei city," Asian Pacific Journal of Allergy and Immunology, vol. 28, no. 1, pp. 1-6, 2010

[27] G. Ben Salah, F. Kallabi, S. Maatoug et al., "Polymorphisms of glutathione S-transferases M1, T1, P1 and A1 genes in the Tunisian population: An intra and interethnic comparative approach," Gene, vol. 498, no. 2, pp. 317-322, 2012.

[28] A. M. Fitzpatrick, D. P. Jones, and L. A. S. Brown, "Glutathione redox control of asthma: from molecular mechanisms to therapeutic opportunities," Antioxidants and Redox Signaling, vol. 17, no. 2, pp. 375-408, 2012.

[29] A. M. Fitzpatrick, S. T. Stephenson, G. R. Hadley et al., “Thiol redox disturbances in children with severe asthma are associated with posttranslational modification of the transcription factor nuclear factor (erythroid-derived 2)-like 2," Journal of Allergy and Clinical Immunology, vol. 127, no. 6, pp. 1604-1611, 2011.

[30] C. Sackesen, H. Ercan, E. Dizdar et al., "A comprehensive evaluation of the enzymatic and nonenzymatic antioxidant systems in childhood asthma," Journal of Allergy and Clinical Immunology, vol. 122, no. 1, pp. 78-85, 2008.

[31] K. R. Shanmugasundaram, S. S. Kumar, and S. Rajajee, "Excessive free radical generation in the blood of children suffering from asthma," Clinica Chimica Acta, vol. 305, no. 1-2, pp. 107$114,2001$.

[32] W. Wu, D. B. Peden, R. McConnell, S. Fruin, and D. DiazSanchez, "Glutathione-S-transferase M1 regulation of diesel exhaust particle-induced pro-inflammatory mediator expression in normal human bronchial epithelial cells," Particle and Fibre Toxicology, vol. 9, article 31, 2012.
[33] A. J. Rogers, C. Brasch-Andersen, I. Ionita-Laza et al., "The interaction of glutathione S-transferase M1-null variants with tobacco smoke exposure and the development of childhood asthma," Clinical and Experimental Allergy, vol. 39, no. 11, pp. 1721-1729, 2009.

[34] J. C. W. Mak, S. P. Ho, H. C. M. Leung et al., "Relationship between glutathione S-transferase gene polymorphisms and enzyme activity in Hong Kong Chinese asthmatics," Clinical \& Experimental Allergy, vol. 37, no. 8, pp. 1150-1157, 2007.

[35] A. Hoskins, S. Reiss, P. Wu et al., "Asthmatic airway neutrophilia after allergen challenge is associated with the glutathione $S$ transferase M1 genotype," American Journal of Respiratory and Critical Care Medicine, vol. 187, no. 1, pp. 34-41, 2013.

[36] P. Bhattacharjee, S. Paul, M. Banerjee et al., "Functional compensation of glutathione S-transferase M1 (GSTM1) null by another GST superfamily member, GSTM2," Scientific Reports, vol. 3, article 2704, 2013. 

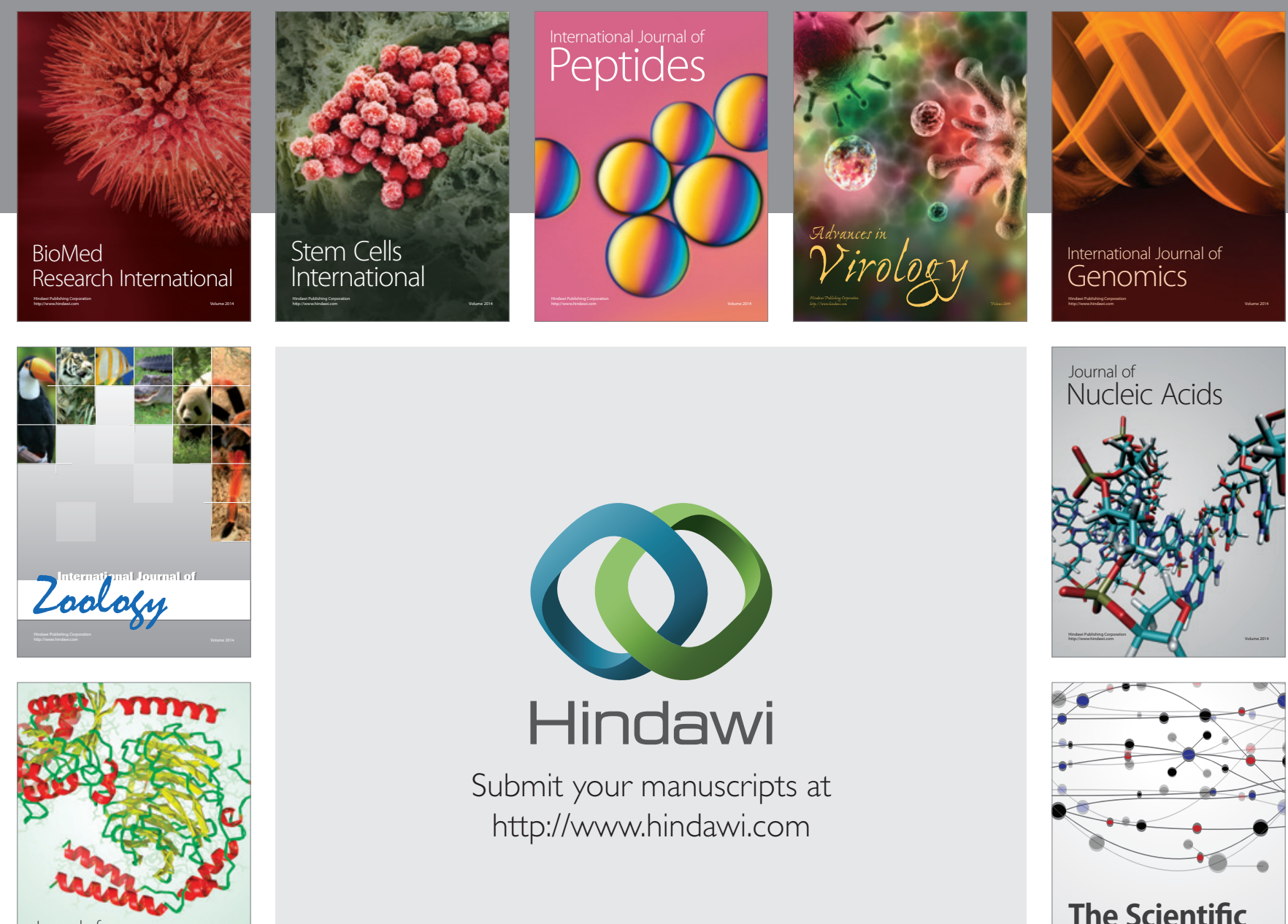

Submit your manuscripts at

http://www.hindawi.com

Journal of
Signal Transduction
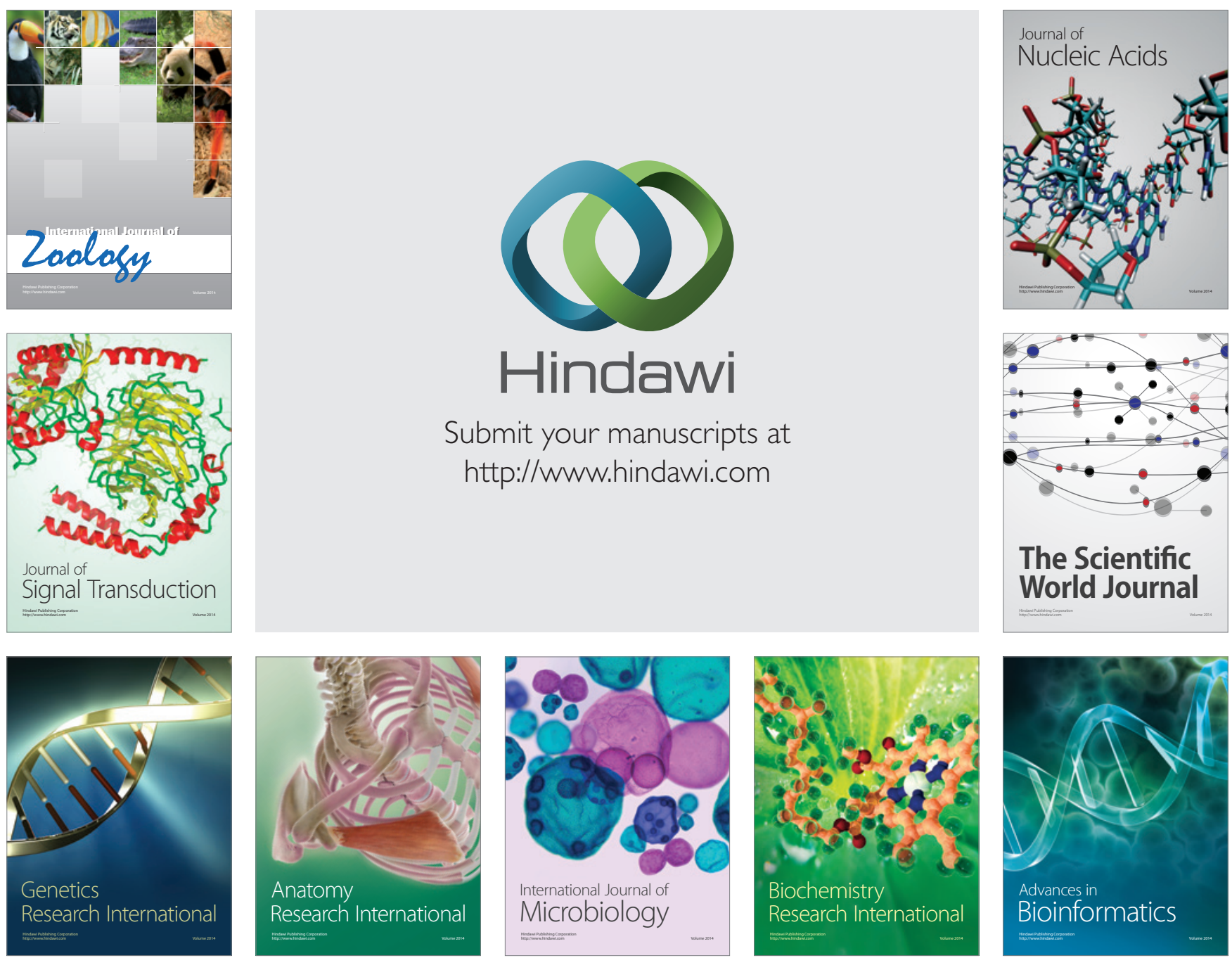

The Scientific World Journal
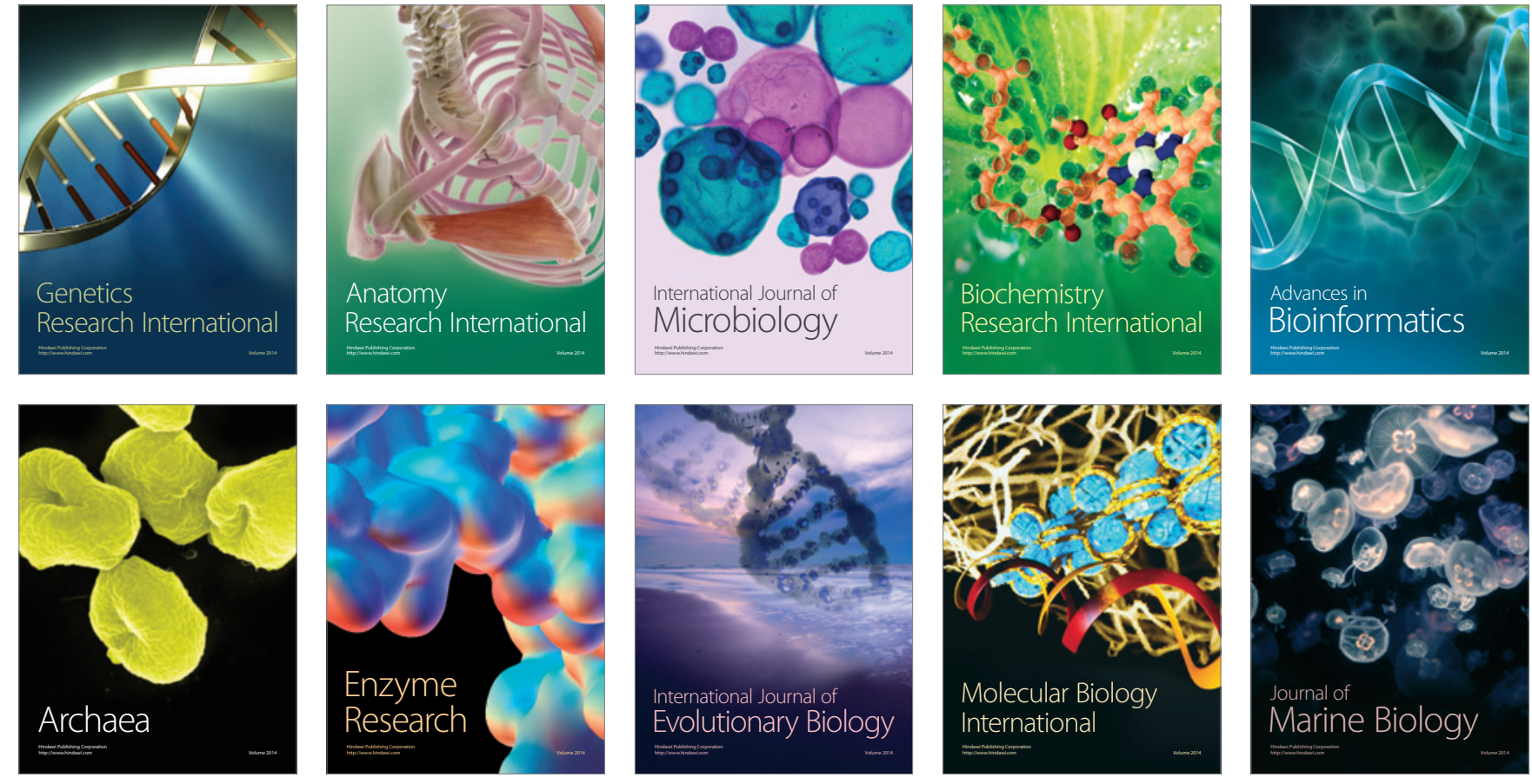